$2^{\circ}$ La virulence des ferments lactiques est très variable. Un nombre plus petit de cellules d'une souche très active aura autant d'effet qu'un grand nombre de cellules d'une souche peu virulente.

Nous avons démontré tout récemment qu'à l'álpage, la boue qui se trouve autour des chalets cantient des bacilles butyriques en grand nombre. Par l'intermédiaire des pieds des animaux et de la couche totalèment dépourvue de litière, ces bacilles s'attachent au pelage de la vache et tombent dans le lait lors de la traite. Ils provoquent alors des boursouflures ou " cuites" et dans les cas graves le gonflement butyrique dans sa forme classique.

\title{
Conclusions
}

Le nombre des bactéries joue un rôle très important dans le lait pour la préparation du fromage de Gruyère ou d'Emmental. Les bactéries nuisibles sont plus nuisibles lorsqu'elles sont plus nombreuses et les bactéries utiles sont nuisibles lorsqu'elles sont trop nombreuses.

Il existe cependant des espèces de bactéries nuisibles quel que soit leur nombre ou à peu près. Nous les nommons bactéries inconditionnellement nuisibles. Les deux espèces reconnues comme telles sont le Bac. amylobacter, l'agent du gonflement en cave, et le Bact. proteolyticum, l'agent de la fermentation secondaire puante.

A l'exception des Bact. coli et aerogenes quand ils provoquent des mammites, les bactéries pathogènes ne sont pas susceptibles de causer directement des troubles de fabrication lorsqu'on prépare du fromage de Gruyère ou d'Emmental.

\section{LA DÉTERIMINATION DU pH DES CASÉINES LACTIQUES}

par

JEAN PIEN

Ingénieur-Chimiste (I. C. R.)

Docteur ès Sciences.

Directeur des Laboratoires des

Fermiers Réunis.

\section{WEISSMANN \\ Chimiste}

des Laboratoires des

Fermiers Réunis.

Nous avons montré au cours d'études antérieures ( $\left.\begin{array}{lll}1 & \grave{a} & 7\end{array}\right)$ comment devait s'effectuer le contrôle chimique des caséines lactiques, comment il convenait d'interpréter les résultats de ce contrôle et quelles doivent être les normes des produits de bonne qualité.

La caséine lactique devant être uniquement constituée par de la protéine pure obtenue par la déminéralisation des phospho- 
caséinates du lait sous l'influence de l'acidification lactique, le contrôle chimique se propose de mettre en évidence à la fois le taux de cette déminéralisation qui doit toujours être aussi grande que possible et l'acidité libre, extérieure à celle de la protéine, qui, traduisant uniquement la perfection des lavages, devrait être toujours nulle ou très faible.

En d'autres termes on demande à la fermentation lactique d'être assez intense et surtout assez prolongée pour que la déminéralisation soit totale et on exige du lavage qu'il soit assez parfait pour débarrasser le produit des sels de chaux séparés de la protéine et de l'acide lactique en excès.

L'analyse chimique détaillée, telle que nous l'avons mise au point et décrite dans nos études précédentes, permet d'instituer et d'interpréter, entre autres ehoses, le contrôle de ces deux points précis.

Or, un pas nouveau doit être franchi dans cette voie : l'examen du $p \mathbf{H}$ des caséines lactiques semble pouvoir nous apporter des critères nouveaux destinés à compléter les données de l'analyse chimique.

Mais il convient d'abord de bien s'entendre sur le sens de l'expression " $p H$ d'une caséine». Ainsi que l'a très justement rappelé M. R. DRU, dans une étude récente [8], la caséine étant un protide insoluble dans l'eau, elle ne saurait donner naissance à une solution aqueuse ionisée.

La détermination du $p H$ dans le cas des caséines ne peut porter que sur une macération aqueuse de ce produit. Et la solution ainsi obtenue renfermera des caséinates si la protéine a été précipitée au-dessus de son point isoélectrique, de l'acide lactique si le lavage a été insuffisant, et même des lactates de caséine si la précipitation a eu lieu à un $p \mathrm{H}$ inférieur à 4,7 .

Le $p H$ de la macération aqueuse renseignera done sur l'importance et la nature de ces ions fixés sur le protide (anions ou cathions) ou restés en mélange avec le produit final, et c'est précisément cet aspect du contrôle qui nous intéresse dans l'appréciation de la qualité des caséines lactiques.

Au point de vue pratique, en effet, si la déminéralisation a été incomplète, e'est-à-dire si la caséine renferme à côté du protide libre des molécules de caséinate, le $p H$ de la macération sera supérieur numériquement à celui du point isoélectrique. $\mathrm{Si}$, la déminéralisation étant complète, le produit fini renferme de l'acide lactique libre par suite de mauvais lavages ou même des radicaux acides combinés aux protides (sels de caséine), le $p H$ sera inférieur à celui du point isoélectrique. Il peut enfin exister des cas intermédiaires où, par suite d'une compensation des deux influences précédentes, le 
$p \mathrm{H}$ de la macération sera plus difficile à interpréter et 'c'est à ce titre d'ailleurs qu'il ne saurait nous dispenser de faire appel à l'analyse chimique.

Nous reviendrons sur cette question de l'interprétation $\mathrm{du} p \mathrm{H}$ des caséines lactiques. Nous avons simplement voulu montrer comment cette notion se rattache directement aux circonstances de la fabrication et nous voulons nous contenter aujourd'hui d'étudier les techniques de sa mesure.

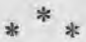

\section{FIXATION DE LA TECHNIQUE DE MACÉRATION}

Nous savons qu'il existe de nombreuses méthodes de détermination du $p H$. Avant d'étudier ces divers procédés pour en déduire celui qui paraît devoir le mieux convenir au cas de la caséine, il convient d'abord de fixer un certain nombre de points de technique indépendants du choix de l'électrode.

La caséine devant être mise au contact de l'eau pour pouvoir donner lieu à une mesure de concentration en ions $\mathrm{H}$, un certain nombre de facteurs interviennent :

Rapport entre le poids de easéine et le volume d'eau.

Durée de contact entre la caséine et l'eau.

Influence du degré de mouture de la caséine.

Il y a lieu également de se demander si, à l'issue de cette macération aqueuse, la mesure doit être effectuée en présence de la caséine elle-même ou seulement sur la phase liquide séparée par filtration.

Ces études préalables ont été effectuées à l'aide de l'électrode hydrogène (électrode basculante de Knight) qui permet des mesures très précises.

\section{Influence du rapport caséine/eau.}

Dans le vase de l'électrode basculante nous avons introduit $50 \mathrm{~cm}^{3}$ d'eau bi-distillée bouillie. Nous y avons ajouté 1 gramme ou 2 gr. 5 , ou 5 grammes de caséine et nous avons effectué la mesure soit immédiatement, soit après un certain temps de macération.

D'une manière générale, comme on pouvait le prévoir par un raisonnement purement théorique, le $p H$ obtenu est d'autant moins acide, que le poids de caséine est plus faible; en diluant, en effet, on diminue la concentration en ions $\mathrm{H}$ et on élève numériquement l'indice $p \mathbf{H}$.

Les écarts ainsi constatés sont donc faibles en général et même parfois nuls entre les prises d'essai de 2 gr. 5 et de 5 grammes. En fait, le rapport caséine-eau ne peut qu'être choisi arbitrairement. 
Poids de caséine mélangée à $50 \mathrm{~cm}^{3}$ d'eau

\begin{tabular}{c|c|c}
\hline 1 gramme & 2 gr. 5 & 5 grammes \\
\cline { 2 - 3 } & 4,83 & 4,80 \\
4,00 & 4,69 & 4,69 \\
5,21 & 5,15 & 5,09 \\
4,80 & 4,76 & 4,74 \\
- & 4,71 & 4,71 \\
\hline
\end{tabular}

Nous avons fixé et nous proposons d'adopter le rapport de 1 à 10 , c'est-à-dire ici 5 grammes de caséine et $50 \mathrm{~cm}^{3}$ d'eau.

\section{Influence de la durée de macération.}

Le mélange caséine-eau étant réalisé dans les conditions précédentes, si l'on effectue la mesure immédiatement à l'électrode basculante, on n'obtient l'équilibre qu'après un temps qui varie de 10 à 20 minutes. Il vient à l'esprit que cet équilibre serait atteint beaucoup plus vite si l'on avait pris soin de laisser la caséine en contact avec l'eau un certain temps avant d'effectuer la mesure.

En réalité cette durée d'équilibre se compose de deux éléments :

a) L'équilibre caséine-eau dont la macération préalable doit réduire le temps.

b) La durée nécessaire à la saturation par l'hydrogène du liquide et de l'électrode elle-même.

Ce deuxième facteur n'existe pas avec les électrodes autres que l'électrode hydrogène. Mais on doit toujours chercher à réduire le premier et on y parvient aisément en laissant la macération se poursuivre quelque temps avant la mesure.

A l'équilibre final, le $p H$ obtenu doit être le même quel que soit le temps de macération. En effet :

\begin{tabular}{c|c|c}
\hline \hline \multicolumn{2}{c}{ Durée des macérations aqueuses } \\
\hline 0 & $1 / 2$ heure & 1 heure \\
\hline 4,83 & 4,83 & 4,83 \\
4,68 & 4,69 & 4,69 \\
5,17 & 5,15 & 5,15 \\
4,84 & 4,81 & 4,81 \\
& & \\
\hline
\end{tabular}

L'intérêt unique de la macération préalable est done de rendre les mesures plus aisées et plus rapides. Cet avantage se manifeste 
assez peu dans le cas de l'emploi de l'électrode hydrogène où la saturation des liquides par l'hydrogène atomisé en quantité suffisante au niveau du platine platiné, demande toujours plusieurs minutes; pendant ce délai l'équilibre caséine-eau peut se poursuivre et la macération préalable ne fait gagner que peu de temps. Mais dans le eas des autres électrodes (quinhydrone; antimoine...), l'équilibre électrique s'établit immédiatement si cette macération préalable de la easéine a été suffisante.

La durée de la macération peut donc être quelconque si l'on fait usage du dispositif de Knight, même dans le cas des électrodes autres que l'électrode hydrogène ; il suffira de s'assurer que l'équilibre électrique ne varie plus dans le temps ou d'attendre qu'il se soit établi parfaitement.

Lorsqu'on a affaire à de la caséine finement pulvérulente (mouture 90), cette macération préalable n'a pas à être prolongée au delà d'une demi-heure. Une macération d'une heure ou de deux heures n'apporte plus aucun progrès. Il reste néanmoins nécessaire de poursuivre la mesure pendant 5 ou 10 minutes dans le cas de l'électrode hydrogène pour les raisons indiquées, alors qu'elle est pratiquement instantanée avec les autres électrodes.

$\mathrm{Si}$, au contraire, on a affaire à des caséines de mouture plus grossière (mouture 30 par exemple) cette macération de 30 minutes n'est plus suffisante. La mesure est très longue et l'obtention d'un équilibre précis reste difficile. En prolongeant la macération préalable jusqu'à deux heures, la conduite de l'essai redevient facile, l'équilibre net, assez lent avee l'électrode $H$., à cause de l'équilibrage de l'électrode, instantané avec les autres.

C'est faute de suivre cette indication qu'on risque avec des moutures grossières d'obtenir des résultats erronés parce qu'on ne s'imposera pas en général de poursuivre l'équilibre électrométrique pendant deux heures; on s'arrêtera prématurément à un pseudoéquilibre électrique que l'on croira être le bon alors que l'équilibre physique caséine-eau n'est pas encore achevé.

\section{$3^{\circ}$ Influence du degré de mouture.}

Nous venons de mentionner l'influence du degré de mouture de la caséine sur la durée de la macération.

En ce qui concerne la valeur de l'indice $p$ H lui-même, ce facteur doit être sans influence d'après ce que nous venons de dire si l'on prend soin de réaliser des macérations préalables suffisantes. Il en est bien ainsi :

Mouture 30

4,64

5,04
Mouture 90

4,64

5,06 


$\begin{array}{cc}\text { Mouture } 30 & \text { Mouture } 90 \\ 4,69 & 4,69 \\ 5,15 & 5,15 \\ 4,78 & 4,76\end{array}$

\section{Influence de la présence de la phase solide.}

Ce que nous avons dit en commençant à l'occasion de la définition du terme " $p H$ des caséines", permet de prévoir que lorsque l'équilibre entre la caséine et l'eau sera obtenu grâce à une macération préalable convenable, la présence de la phase solide ne sera plus nécessaire pour la détermination du $p \mathrm{H}$ de ce liquide de macération. L'expérience montre que cette manière de voir est conforme à la réalité.

Les chiffres suivants ont été obtenus après une macération de deux heures à l'électrode basculante à hydrogène.

\begin{tabular}{c|c}
\hline \multicolumn{2}{c}{ Mesures effectuées } \\
\hline En présence de la caséine & Sur le liquide filtré \\
\hline & \\
4,39 & 4,40 \\
4,18 & 4,18 \\
4,57 & 4,57 \\
4,55 & 4,55 \\
4,81 & 4,81 \\
5,01 & 5,01 \\
4,53 & 4,53 \\
\hline
\end{tabular}

Il suffira donc dans tous les cas d'opérer sur le liquide de macération filtré ou simplement décanté.

\section{En résumé}

La préparation de la macération de caséine s'effectuera de la manière suivante :

Dans un récipient en verre neutre de $100 \mathrm{~cm}^{3}$ bouchant à l'émeri, on introduit $50 \mathrm{~cm}^{3}$ d'eau bi-distillée bouillie et 5 grammes de caséine. On ferme et on agite șoigneusement.

On laisse la macération se poursuivre pendant une demi-heure s'il s'agit d'une caséine de fine mouture et pendant deux heures s'il s'agit d'une mouture grossière. Il n'y a d'ailleurs pas d'inconvénient à adopter deux heures de macération dans tous les cas à condition que le flacon reste bien bouché pendant toute la durée du contact. On aura soin d'agiter de temps à autre.

A l'issue de la macération on laisse reposer et on décante le liquide surnageant dans le vase de l'électrode. 


\section{$*^{*} *$ \\ II. CHOIX DE L'ÉLECTRODE}

Nous avons étudié comparativement : l'électrode à hydrogène, l'électrode à quinhydrone, et l'électrode d'antimoine.

L'électrode à hydrogène est toujours assez longue à équilibrer et elle nécessite une installation un peu compliquée.

L'usage de la quinhydrone n'exige que la saturation préalable du liquide de macération par ce réactif immédiatement avant la mesure - laquelle d'ailleurs est pratiquement instantanée.

L'emploi de l'électrode d'antimoine, grâce aux stylélectrodes Prolabo d'un emploi si facile, n'entraîne aucune manipulation spéciale et fournit également un équilibre électrique instantané.

Il y avait évidemment lieu de se demander si ces trois électrodes fourniraient des indications concordantes.

Voici les résultats obtenus après macérations convenables. Nous y avons joint quelques déterminations colorimétriques effectuées au vert de bromocrésol sur le liquide de macération filtré.

$\begin{array}{cccc}\begin{array}{c}\text { Electrode } \\ \text { hydrogène }\end{array} & \begin{array}{c}\text { Electrode } \\ \text { quinhydrone }\end{array} & \begin{array}{c}\text { Electrode } \\ \text { antimoine }\end{array} & \begin{array}{c}p \mathrm{H} \\ \text { colorimétrique }\end{array} \\ 4,05 & 4,05 & 4,05 & 4,0 \text { fort } \\ 4,39 & 4,35 & 4,36 & 4,4 \text { faible } \\ 4,18 & 4,19 & 4,23 & 4,2 \\ 5,01 & 5,06 & 5,04 & 5,0 \text { faible } \\ 4,53 & 4,55 & 4,48 & 4,4 \text { fort }\end{array}$

Pratiquement ces résultats sont eoncordants surtout entre la quinhydrone et l'hydrogène. On peut donc abandonner pour la pratique $d u$ contrôle industriel l'électrode hydrogène et adopter l'électrode à quinhydrone (1) déjà recommandée par M. DRU [8] dans le cas des caséines-présure. On pourrait aussi se servir de l'électrode d'antimoine qui ne nous a jusqu'à présent jamais causé d'ineonvénients sérieux en dépit des critiques dont elle est l'objet de la part de quelques auteurs.

Quant á la réalisation du montage, elle est des plus simples. On peut faire appel au dispositif préconisé par M. DRU (éleetrode d'Aten légèrement modifiée) ou encore au montage classique avec pont de gélose au $\mathrm{KCl}$ conformément au eroquis ci-joint.

On remarquera que le liquide de macération est introduit dans un petit vase ouvert à l'air libre. L'équilibre électrique étant obtenu instantanément aussi bien dans le cas de la quinhydrone que dans le cas de l'antimoine, les perturbations dues au $\mathrm{CO}^{2}$ atmosphériques ne sont pas à redouter car elles n'ont pas le temps d'intervenir et

(1) En utilisant une petite plaque de platine poli plutôt qu'un fil, ce qui permet d'obtenir une plus grande sensibilité dans la mesure. 


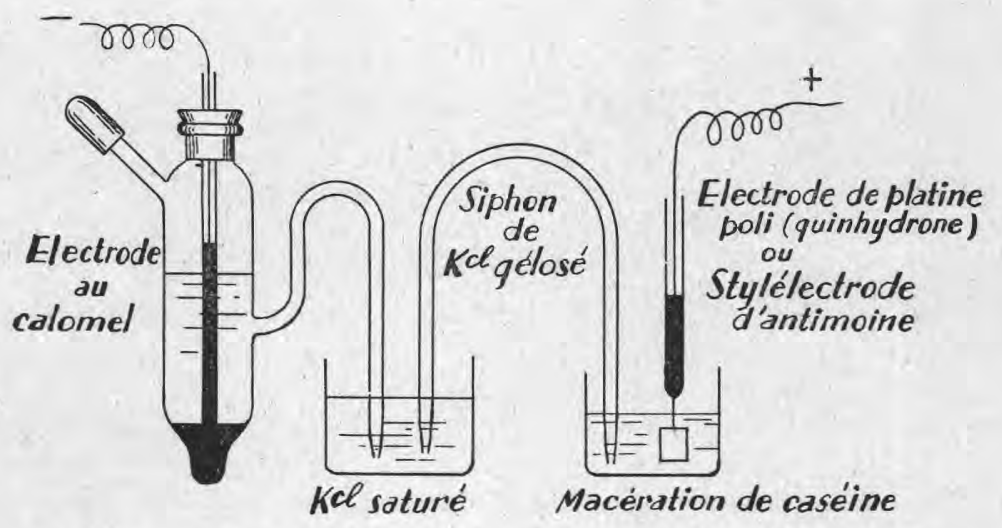

il en résulte une simplification du montage. Cette disposition permet en outre de n'utiliser que quelques centimètres cubes du liquide de macération et de déterminer très rapidement le $p \mathrm{H}$ d'une grande série d'échantillons.

Nous aurons l'occasion de revenir sur cette question pour montrer comment il convient d'interpréter les données fournies par cette technique dans la pratique du contrôle des caséines lactiques.

\title{
BIBLIOGRAPHIE
}

[1] J. Pien. Le Lait, t. XI, 1931, no 110, p. 1038.

[2] J. Pien et M. Bergier, Le Lait, t. XII, 1932, no 117, p. 640.

[3] J. Pien et S. Herschdoerfer. Le Lait, t. XIII, 1933, no 129, p. 1081.

[4] J. Pien et S. Hersohdoerfer. Le Lait, t. XIV, 1934, no 131, p. 30.

[5] J. Pien et M. Bergier. Le Lrit, t. XIV, 1934, no 132 , p. 155 et no 133 , p. 248.

[6] J. Pien, R. Martin et M. Bergier. Le Lait, t. XIV, 1934, no 134, p. 356 ; $\mathrm{n}^{\circ} 135$, p. $483 ; \mathrm{n}^{\circ} 136$, p. 582 .

[7] J. Pien, R. Martin et S. Herschdoerfer. Le Lait, t. XIV, 1934, no 137 , p. 709 .

[8] R. Dru. Le contrôle de la caséine-présure. XVII ${ }^{\mathrm{e}}$ Congrès de Chimie industrielle, Paris, 26 septembre et 3 octobre 1937.

\section{L'ORGANISATION DU CONTRÔLE HYGIÉNIQUE DU LAIT}

\author{
par le
}

\section{Dr G. BARTHÉLEMI}

Médecin-hygiéniste. M.R. San. I.

Dans le programme de rénovation du marché du lait ou pour mieux dire, dans la solution de cette importante question que constitue le problème du lait, il faut réserver une large part à 